\title{
Flow Separation Study in Stiff Ovalized Rocket Nozzles, Part II: Numerical Approach
}

\author{
Sebastian Jack* \\ German Aerospace Center (DLR), Braunschweig, Germany \\ Chloé Génin ${ }^{\dagger}$ \\ German Aerospace Center (DLR), Lampoldshausen, Germany
}

\begin{abstract}
During the transient startup and shutdown process of a rocket engine high side loads occur, due to unsymmetrical flow patterns. The resulting deformation, and its retroactive effect onto the internal flow, excite the nozzle structure and can lead to its fatal damage. Flow separation at the nozzle wall amplifies the deformation. To investigate and predict the effects as well as the underlying mechanism that cause these phenomena, the flow characteristics in ovalized nozzles are investigated in the DLR project ProTAU. In this paper CFD results of ovalized TIC nozzles are presented and compared to experimental data, to validate the used numerical method. The presented results show good overall agreement for the relevant regimes, with a tendency to slightly overpredict the separation position. In the consecutive step the method will be extended to simulate the coupled problem of flow structure interaction.
\end{abstract}

\section{Nomenclature}

$\alpha$

circumferential angle

(see figure 3a for measurements)

$\varepsilon \quad$ numerical eccentricity

$l \quad$ nozzle length

Ma Mach number

$p \quad$ static pressure

$R \quad$ local radius

$v \quad$ velocity

$X \quad$ coordinate in main flow direction

Subscript

0 total condition

a ambient condition

$c \quad$ cutting plane

def deformation

des design state

div divergent nozzle part

$e \quad$ nozzle exit plane

$M D \quad$ Mach disk

sep separation

$t$ tangential

th nozzle throat
Abbreviations

NPR nozzle pressure ratio

RSM Reynolds stress turbulence model

SA Spalart Allmaras turbulence model

TIC truncated ideal contour

\footnotetext{
* Research Assistant, Institute of Aerodynamics and Flow Technology, Lilienthalplatz 7, 38108 Braunschweig, AIAA Member

${ }^{\dagger}$ Research Scientist, Institute of Space Propulsion, Langer Grund, 74239 Lampoldshausen, AIAA Member
} 


\section{Introduction}

Asymmetrical and transient effects, such as combustion instabilities, the unsteady flow around the rocket body, or material imperfections, deform the structure of a rocket engine nozzle during its operation. When the nozzle works around its design point, i.e. the nozzle pressure ratio $\left(\mathrm{NPR}=p_{0} / p_{a}\right)$ is high enough to ensure a supersonic flow attached to the wall down to the exit plane, a deformation of the nozzle wall leads to a change in the wall pressure distribution counteracting the local displacement. The interaction between the nozzles inner flow and its structure damps the systems excitation. For a highly overexpanded state, where the internal flow faces a strong positive pressure gradient, it separates from the nozzle wall. Downstream the separation line the wall pressure increases to the magnitude of the ambient pressure. In this case an initial deformation of the nozzle wall leads to a displacement of the separation line which implies forces that further increase the deformation, the coupled system is excited by the flow-structure-interaction. For a detailed description of the ovalization mechanism and side load generation in separated nozzle flows that initiate this process see. ${ }^{1-3}$ Since during the highly transient startup and shutdown process of rocket engines highly overexpanded flows necessarily occur and an excitation of the nozzles eigenmodes can lead to its fatal damage, this problem is relevant for the nozzle dimensioning process. The flow characteristics in ovalized rocket nozzles and the interacting influence on the nozzle structure is therefore investigated within the framework of the DLR internal cooperation program ProTAU.

\section{Methodology}

Flow separation in deformed rocket nozzles has been investigated in different studies by various authors. For a detailed literature overview see the publications by Génin et. al., ${ }^{1}$ or Hadjadj and Onofri. ${ }^{4}$ Most of these studies focused on numerical simulations since the experimental measurement of the flow-structureinteraction in a supersonic rocket nozzle is very challenging. To be able to investigate the underlying phenomena, the complexity of the coupled process is therefore increased step by step in the present study. At first, three rotation-symmetric nozzles have been designed as baseline geometries. These geometries have then been deformed by generic functions to stiff ovalized nozzles. In the subsequent future step, the instationary coupled system of flow and deforming structure will be investigated. For each project phase numerical and experimental studies are carried out to collect validated data.

\section{II.A. Nozzle Geometries}

As baseline geometries three rotation-symmetric nozzles have been designed to evaluate the accuracy of the simulation method and the effect of the nozzle design parameters. Truncated ideal contour (TIC) nozzles have been chosen for this study due to their relatively simple shock pattern which also influences the separation behavior. For comparability all designed geometries have a throat radius of $R_{\mathrm{th}}=10 \mathrm{~mm}$ and are designed to be full flowing at a NPR of 50. The nozzles differ in their design Mach number $M a_{\text {des }}(4.8,5.3$, and 5.8) and therefore the length of the divergent part $l_{d i v}$, area ratio $\left(R_{e} / R_{\mathrm{th}}\right)^{2}$ and exit wall angle. The contours are named by their design Mach number: TIC-48, TIC-53, and TIC-58. Table 1 shows an overview of the undeformed nozzle's parameters.

Table 1: Undeformed nozzle geometry parameters

\begin{tabular}{ccc} 
geometry & $M a_{\text {des }}$ & $\left(R_{e} / R_{\mathrm{th}}\right)^{2}$ \\
\hline TIC-48 & 4.8 & 16.9 \\
TIC-53 & 5.3 & 18.5 \\
TIC-58 & 5.8 & 19.3
\end{tabular}


To examine the effect of ovalization on the flow, a set of seven deformed geometries was created out of the baseline nozzles by varying the amplitude $\Delta R_{\text {def,max }} / R_{e}$, starting point $\left(R / R_{\mathrm{th}}\right)^{2}{ }_{\text {def,start }}$, and the applied generic function of the deformation. The range of these parameters were exaggerated compared to the deformation behavior of realistic rocket engine nozzles in order to achieve sufficiently measureable effects. Table 2 shows the varied parameters and the nozzle's numbering that will be used in all figures below. The flow in all geometries has been simulated in a preliminary study. On the basis of this study, geometries 2 to 4 (shown in figure 1) were then chosen to be manufactured and investigated experimentally.

Table 2: Deformed nozzle geometry parameters

\begin{tabular}{ccccc} 
geometry & reference nozzle & $\Delta R_{\text {def, } \max } / R_{e}$ & $\left(R / R_{\mathrm{th}}\right)^{2}$ def,start & function \\
\hline 1 & TIC-48 & $5 \%$ & 5 & parabola \\
2 & TIC-48 & $10 \%$ & 5 & parabola \\
3 & TIC-48 & $15 \%$ & 5 & parabola \\
4 & TIC-53 & $10 \%$ & 1 & parabola \\
5 & TIC-53 & $10 \%$ & 5 & linear \\
6 & TIC-53 & $10 \%$ & 5 & parabola \\
7 & TIC-58 & $10 \%$ & 5 & parabola
\end{tabular}

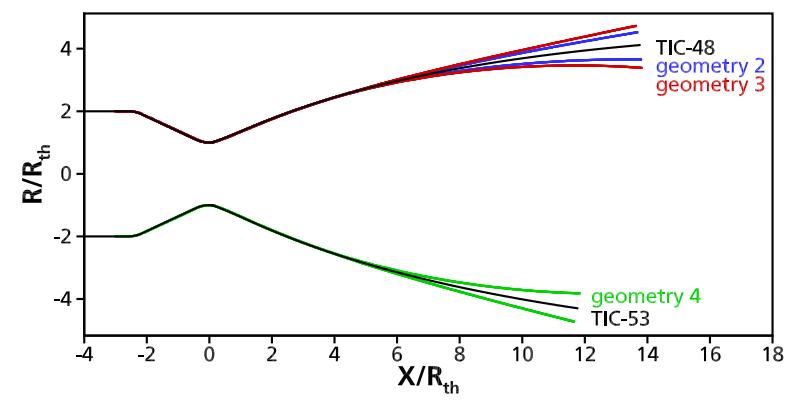

Figure 1: Contours of undeformed nozzles TIC-48 and TIC-53 and the derived deformed geometries 2,3 , and 4 at their minor and major axis.

\section{II.B. Experimental Setup}

All experimental investigations were performed at the P6.2 test facility at DLR Lampoldshausen using dry nitrogen. In order to ensure stiff geometries, the nozzles have been manufactured of acrylic glass with a wall thickness of $8 \mathrm{~mm}$. Each nozzle was equipped with various pressure sensors along five axial lines within one quadrant, including the minor $\left(\alpha=0^{\circ}\right)$ and major semi-axis $\left(\alpha=90^{\circ}\right)$ and the undeformed section $\left(\alpha=45^{\circ}\right)$. The resulting angular resolution is $\Delta \alpha=22.5^{\circ}$, with 19 sensors in each line. Additional to the pressure measurements a schlieren optic was used to visualize the shock system in the nozzle's exhaust flow. By rotating the nozzle model between the test runs a three-dimensional image of the shock system could be reconstructed. Figure 2 shows geometry four mounted on the test bench. For a detailed description of the experimental setup, the test environment and the measurement equipment see the experimental part of this study. ${ }^{5}$

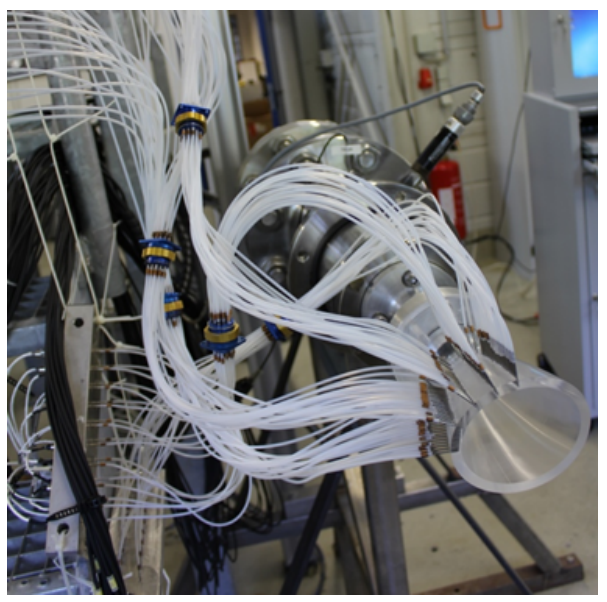

Figure 2: Geometry 4 at horizontal test rig P6.2. 


\section{II.C. Numerical Method}

The presented simulations were all performed using the DLR finite-volume solver TAU. The stationary Reynolds-averaged Navier Stokes equations were solved using Nitrogen as perfect gas as a fluid model. To close the equation system either the original formulation of the Spalart Allmaras (SA) or a Reynolds stress (RSM) turbulence model was used. The used RSM consists of the Wilcox stress- $\omega$ model as re-distribution model, the isotropic model by Rotta as dissipation model, a diffusion model based on the generalized gradient diffusion hypothesis, and the necessary length scale computed on basis of the Menter baseline $\omega$-equation. All calculations have been performed on hybrid grids of a quarter section $\left(90^{\circ}\right.$ opening angle) with symmetry planes of the respective geometry. Automatic mesh adaptation was used to ensure grid convergence.

The non-rotation-symmetric shape of the ovalized nozzles leads to a deformation of both, the separation line and the shock system in the nozzles exhaust flow. To measure and quantify the deformations influence onto these flow effects they were automatically extracted from the flow field and appropriate functions were fitted to the numerical data. The parameters of these fits could then be compared to the deformation input parameters. Along the nozzles inner surface the wall pressure gradient was analyzed to follow the separation line. The distorted sine function shown in equation (1) was then fitted to the resulting raw data.

$$
X_{\text {sep }}=\hat{X}_{\text {sep }} \cdot \sin \left(2 \alpha+\frac{\pi}{2}+c \cdot \sin (2 \alpha-\pi)\right)+\bar{X}_{\text {sep }}
$$

To detect the Mach disk and quantify its curvature the local maxima of the density gradient has been analyzed. Since the Mach disk in an undeformed nozzle is bended to a saddle shape by the ovalization, a hyperbolic paraboloid in the cross section and another sine function in the radial section have been fitted to the raw data (see equations (2) and (3)).

$$
\begin{aligned}
X_{M D} & =\hat{X}_{M D} \cdot \sin \left(2 \alpha+\frac{\pi}{2}\right)+\bar{X}_{M D} \\
1 & =\left(\frac{Z_{M D}}{R_{M D, \text { max }}}\right)^{2}+\left(\frac{Y_{M D}}{R_{M D, \text { min }}}\right)^{2}
\end{aligned}
$$

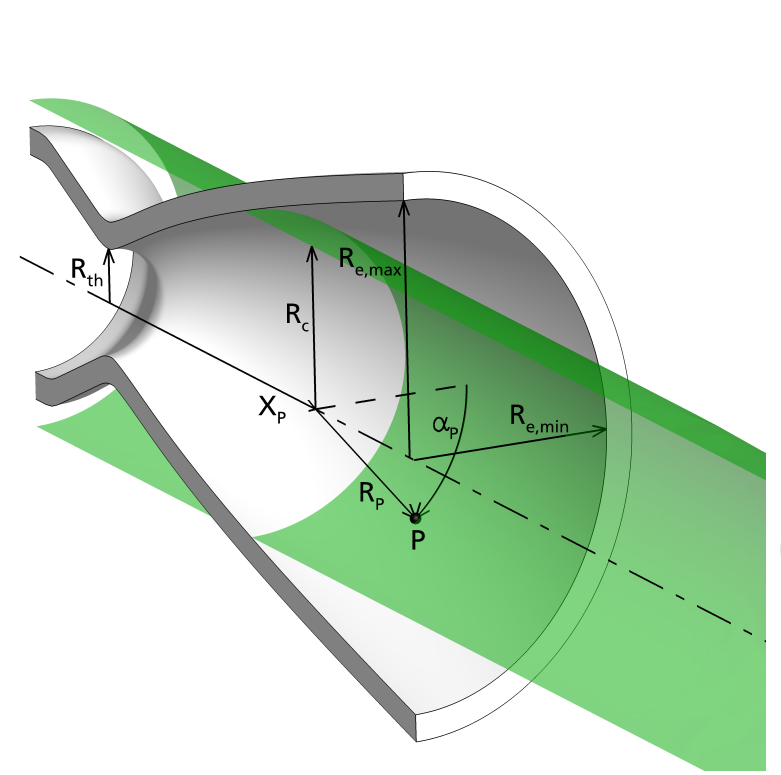

(a)

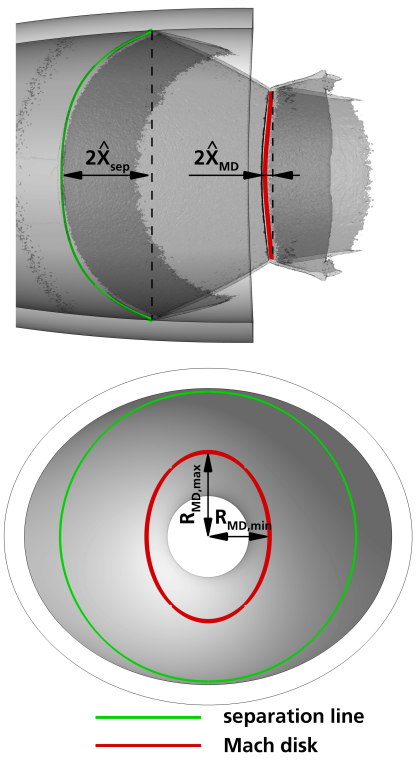

(b)

Figure 3: (a) Dimensioning of the nozzle properties shown at a half model of geometry 4 including a cylindrical cutting plane at radius $R_{c}$ (green). Point $\mathbf{P}$ is just an example to illustrate the references of $\alpha, R$ and $X$.

(b) Typical flow pattern displayed by iso-surfaces of the density gradient together with fitted functions for separation line (green) and Mach disk (red) in geometry 2 for NPR 35. 


\section{Results}

Figure 4 shows the wall pressure for geometry four at a cutting angle $\alpha=67.5^{\circ}$ for NPR $=20$ and $\mathrm{NPR}=40$. The black lines in the figure represent the wall pressure distribution calculated by three different methods. The solid and dashed lines are results of CFD calculations using the RSM and SA turbulence model. The dot-and-dash line shows the results of an analytical approximation. For this approximation the nozzle flow was considered isentropic and one dimensional in each cross section of the nozzle. The wall pressure can then be calculated from the local nozzle radius and the isentropic flow equations. The pressure was then corrected with data of the full flowing undeformed nozzle. This leads to a very fast analytical approximation of the full flowing deformed nozzle. In the supersonic region of the nozzle the numerical results, including the

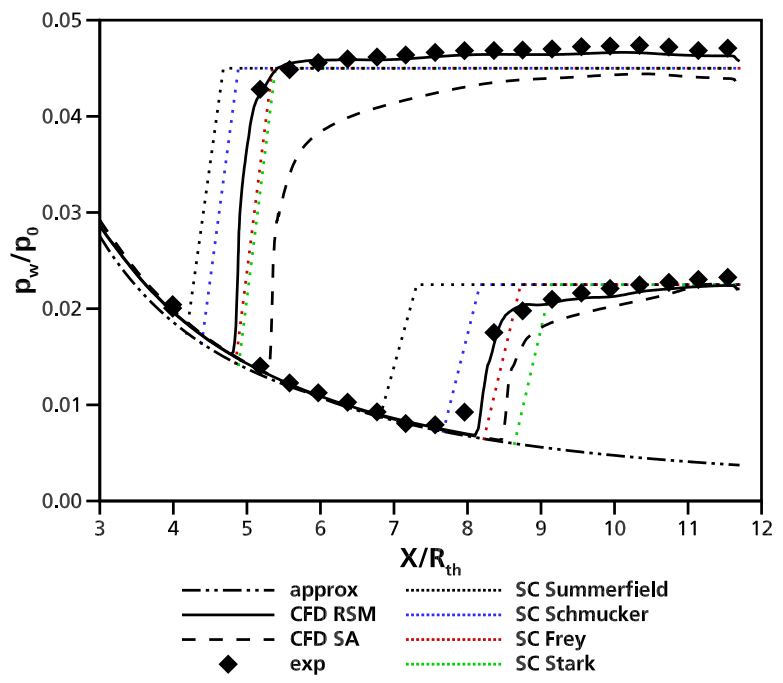

Figure 4: Wall pressure distribution for geometry 4 at a cutting angle of $\alpha=67.5^{\circ}$ at NPR $=20$ and NPR $=40$, calculated using the Spalart Allmaras (SA) turbulence model, the Reynolds stress model (RSM) and an analytical approximation (approx).

calculations using the Spalart Allmaras turbulence model and even those of the analytical approximation, fit the experimental data very well. However the separation position predicted by the SA model calculations lies significantly downstream the one obtained from the experiments. Additionally the wall pressure in the recirculation area is predicted too low. Both of these effects are in good agreement with the results obtained from the investigation of the undeformed geometries and earlier numerical studies. ${ }^{6,7}$ The use of a Reynolds stress turbulence model significantly enhanced the prediction accuracy, especially concerning the pressure in the recirculation area downstream the separation position. A variety of models were developed to predict the separation position in supersonic nozzles, especially for the application in rocket engines. Four of these models were applied to the above mentioned approximation to estimate the separation position. The chosen models are the ones developed by Summerfield ${ }^{8}$ and Schmucker, ${ }^{9}$ because of their wide application, as well as those by Frey ${ }^{10}$ and Stark, ${ }^{11}$ because these authors developed their models on the wide basis of data about nozzles similar to those used in this study. For the application of the criterion by Frey a constant plateau to ambient pressure ratio of $p_{p} / p_{a}=0.9$ was chosen. The separation positions calculated by the above mentioned models are shown as dotted lines in figure 4. While the criteria by Summerfield and Schmucker tend to underpredict the separation position, the criterion by Stark (for lower NPR) and especially the one by Frey give a good estimation for the separation point along this axial section.

Figure 5 shows the parameters of the functions fitted to the separation line (upper plots) and the Mach disk (lower plots) that represent their position and shape for geometry four. It can be seen that the CFD results calculated using the SA model yield a mean separation position $\bar{X}_{\text {sep }}$ that lies downstream the measured data and that the deviation falls with rising NPR. The use of a RSM model reduces this general deviation and leads to a correct reproduction of the pressure dependency. The mean separation position is also very well predicted by Frey's separation criterion. For the separation line curvature, that is illustrated by the separation fit amplitude $\hat{X}_{\text {sep }}$, the differences in the prediction accuracy are emphasized. While the RSM calculations again predict the correct gradient with a slight overprediction of the amplitude, all of 
the applied separation criteria fail to reproduce its magnitude and trend. This effect is mainly caused by the interaction of the separation shock with the vortex system located at the nozzle end. With rising NPR the separation lines moves downstream leading to its stronger deformation. For NPR $>40$ the separation reaches the nozzle exit and the deformation amplitude decreases.
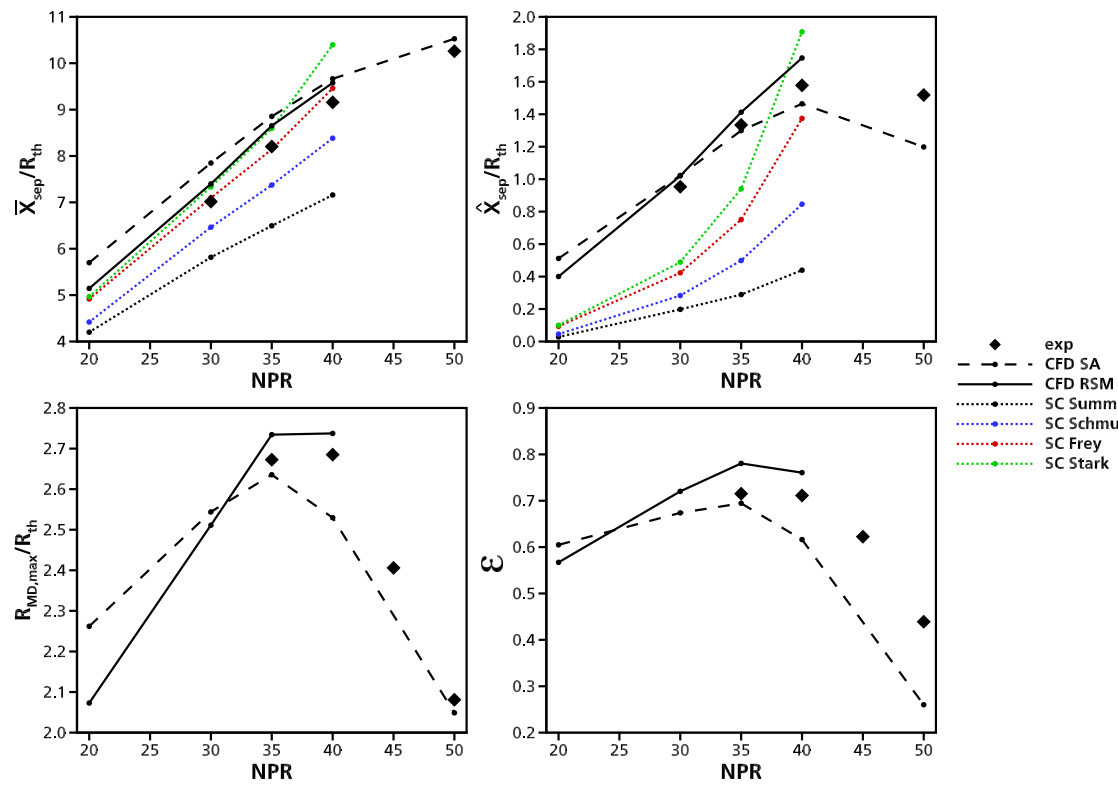

............ SC Summerfie

............. SC Schmucker

Figure 5: Parameters of fitted functions for geometry 4.

Size and shape of the distorted Mach disk are being represented by the large semi axes $R_{M D, \max }$ and the numerical eccentricity $\varepsilon$, respectively. The lower plots in figure 5 show that the used turbulence model has a great influence on these parameters. Although the experimental data for the shock system is restricted to NPR $\geq 35$, due to the optical measurement technique that can only capture shock outside the nozzle, the results indicate a trend similar to the separation data. The simulation results, especially those obtained by the RSM model, reproduce the correct trend for the pressure influence.

The tangential velocity, which is zero for a perfectly rotational symmetric nozzle flow, is shown in figure 6. It is plotted on the cylindrical cutting plane for three different cutting radii $R_{C}$ as shown in figure $3 \mathrm{a}$. It can be seen that in the flow upstream the separation shock the tangential velocity is zero. The flow in this region can be modelled using simple axisymmetric methods. The tangential velocity in the flow downstream the oblique shock however rises up to an order of $100 \mathrm{~m} / \mathrm{s}$ which is around $40 \%$ of the velocity in main flow direction $\left(v_{X}\right)$ in this region. This crossflow leads to the aforementioned effect of an earlier separation that is present in the wall pressure distributions for all geometries.

The effects and deviations described for geometry four were observed for all three measured geometries.
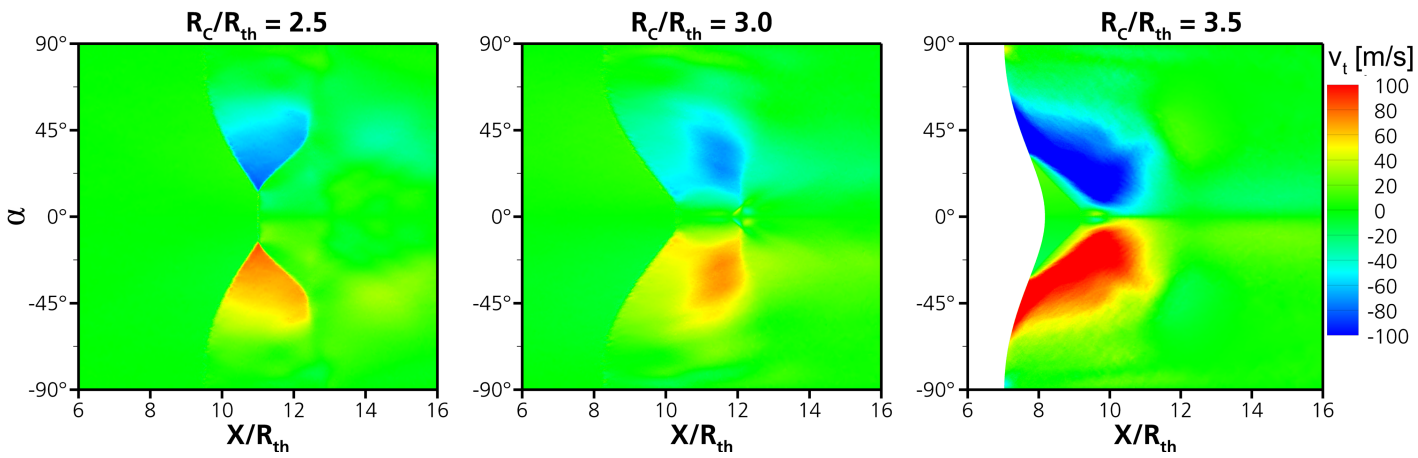

Figure 6: Tangential velocity at cylindrical surface extracted from CFD domain of geometry 4 at radius $R_{c}$. 
Figures 7, 8, and 9 show the wall pressure data for all measurement positions and a range of pressure ratios from 20 to 40 compared to CFD data using the RSM model. The plots show a good agreement for the supersonic flow and the distribution downstream the separation position. The separation position itself is predicted slightly too far downstream for all geometries. This deviation is higher for geometry two and especially geometry three, which is caused by the slim shape of their baseline geometry TIC-48 and emphasized by the higher deformation amplitude of geometry three.

For geometry four the red line in figure 9 at $\alpha=45^{\circ}$ shows the pressure distribution of the undeformed nozzle. At this circumferential angle the nozzle was not deformed. The different separation positions again show the influence of the tangential flow component in the recirculation area as stated above.
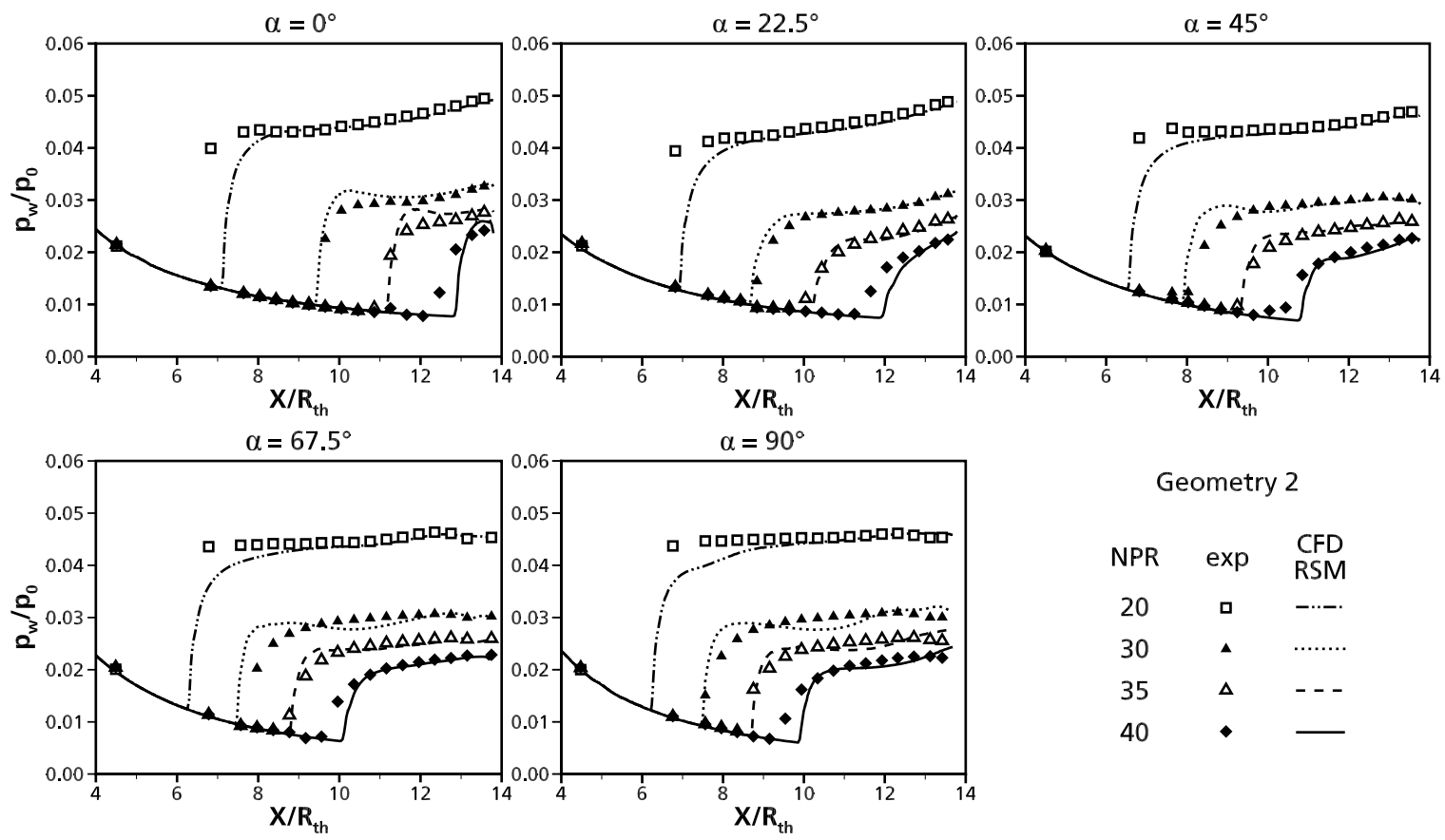

Geometry 2

Figure 7: Wall pressure obtained by Simulation and Experiment for deformed geometry 2 at different cutting angles $\alpha$. 

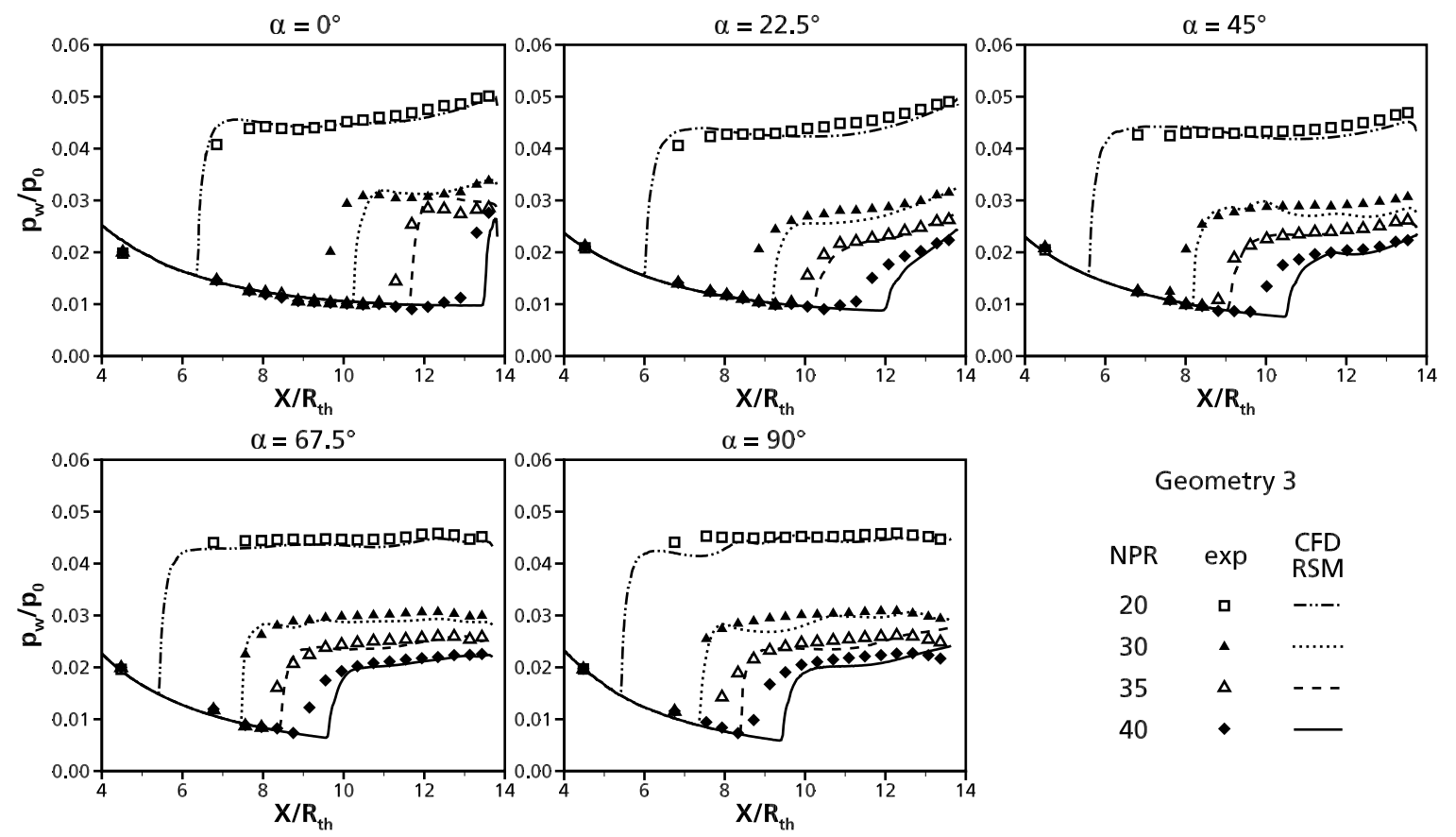

Geometry 3

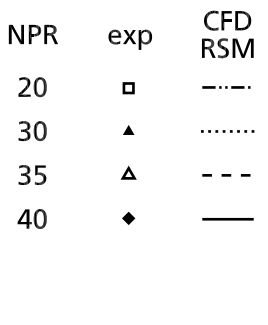

Figure 8: Wall pressure obtained by Simulation and Experiment for deformed geometry 3 at different cutting angles $\alpha$.
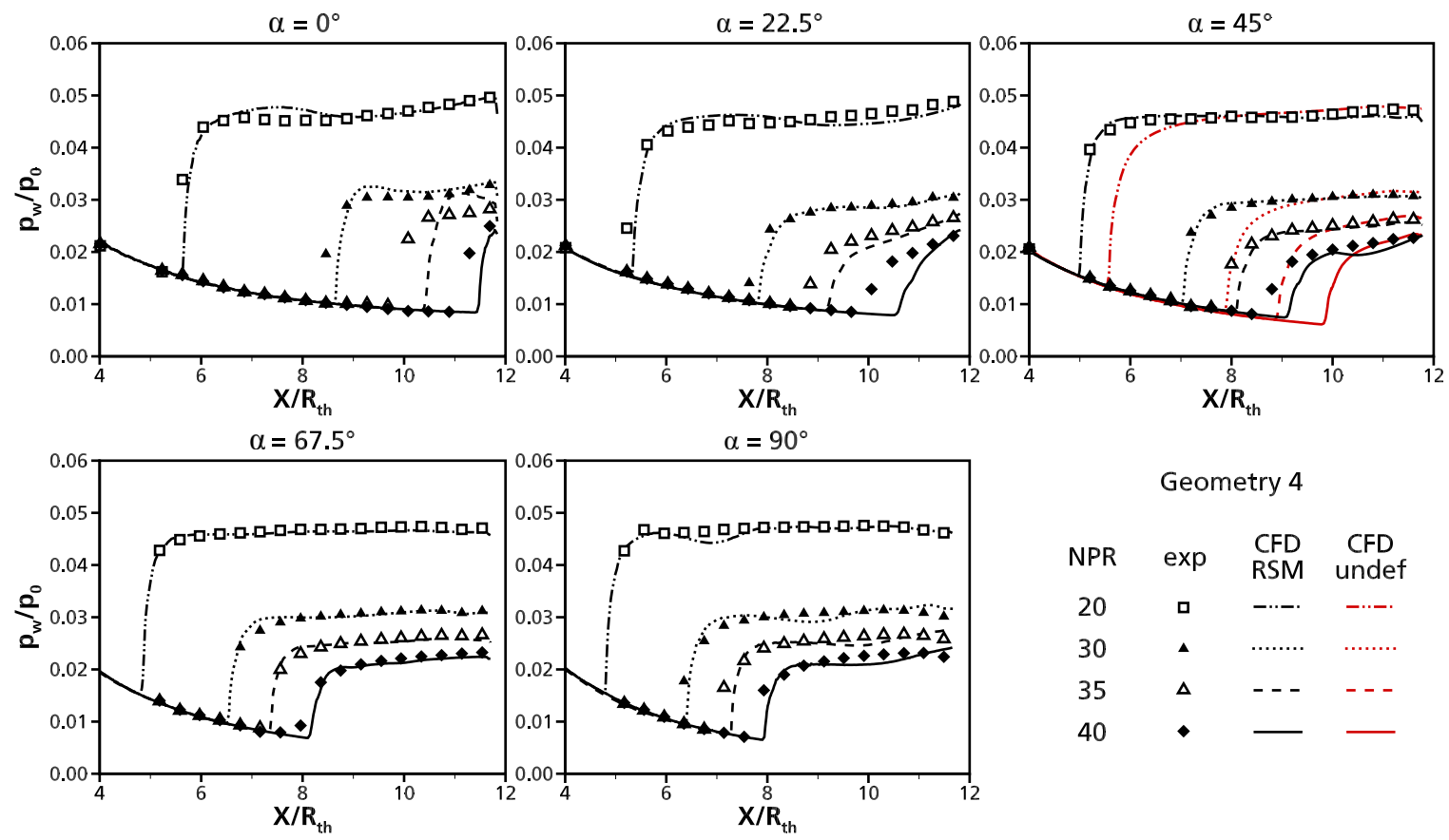

Figure 9: Wall pressure obtained by Simulation and Experiment for deformed geometry 4 at different cutting angles $\alpha$. 


\section{Conclusion}

Earlier studies for the rotation-symmetric baseline nozzles carried out in the framework of the ProTAU project show a good overall agreement for CFD results and experimental data when using a Reynolds stress model, with a tendency to slightly overpredict the separation position. The consecutive investigation of ovalized nozzles, discussed in the presented work, shows comparable results. For low and moderate NPR, when the separation line does not interact with the nozzle exit, the separation position and wall pressure distribution can be predicted reliably by the presented numerical method. The deviation to the experimental results rises for small contour angles (i.e. slim nozzles). For practical application, where the deformation amplitude is approximately $3 \%$ of the nozzle exit Radius $R_{e}$, the used method is capable to calculate realistic wall pressure data for the critical phase in which a large recirculation area occurs. The influence of the deformation and the deviations of the numerical and experimental method will be investigated in the following studies, that will focus on the coupling between the nozzle flow and the deformation behavior of its structure.

\section{References}

\footnotetext{
${ }^{1}$ Génin, C., Stark, R., Jack, S., Flow Separation in Out-of-Round Nozzles, a Numerical and Experimental Study, Progress in Flight Physics, Vol. 7, Springer, 2015.

${ }^{2}$ Damgaard, T., Östlund, J., Frey, M., Side-Load Phenomena in Highly Overexpanded Rocket Nozzles, Journal of Propulsion and Power, Vol. 20(4), AIAA, 2004.

${ }^{3}$ Östlund, J., Flow Processes in Rocket Engine Nozzles with Focus on Flow Separation and Side-Loads, 2002.

${ }^{4}$ Hadjadj, A., Onofri, M., Nozzle Flow Separation, Shock Waves, Vol. 19(3), Springer, 2009.

${ }^{5}$ Génin, C., Jack, S., Flow Separation Study in Stiff Ovalized Rocket Nozzles, Part I: Experimental Approach, Joint Propulsion Conference, Orlando, 2015.

${ }^{6}$ Stark, R., Hagemann, G., Current Status of Numerical Flow Prediction for Separated Nozzle Flows, 2nd European Conference for Aerospace Sciences (EUCASS), Brussels, 2007.

${ }^{7}$ Wagner, B., Karl, S., Hagemann, G., Test Case 1a: Short Nozzle Under Separated Flow Condition, a Numerical Investigation with the DLR TAU-Code, FSCD-ATAC Workshop, 2006.

${ }^{8}$ Summerfield, M., Foster, C. R., Swan, W. C., Flow Separation in Overexpanded Supersonic Exhaust Nozzles, Jet propulsion, Vol. 24(5), AIAA, 1954.

${ }^{9}$ Schmucker, R. H., Strömungsvorgänge beim Betrieb überexpandierter Düsen chemischer Raketentriebwerke, Teil 1: Strömungsablösung, 1973.

${ }^{10}$ Frey, M., Behandlung von Strömungsproblemen in Raketendüsen bei Überexpansion, 2001.

${ }^{11}$ Stark, R., Experimental Study of Boundary Layer Separation in Truncated Ideal Contour Nozzles, Shock Waves, Vol. 19, Springer, 2009.

${ }^{12}$ Jack, S., Génin, C., Numerical and Experimental Investigation of Flow Separation in Ovalized Nozzles, Space Propulsion Conference, Cologne, 2014.
} 